\title{
Alopecia Areata Treatment Patterns, Healthcare Resource Utilization, and Comorbidities in the US Population Using Insurance Claims
}

\author{
Maryanne Senna · Justin Ko · Antonella Tosti · Emily Edson-Heredia • \\ D. Christian Fenske · Amy K. Ellinwood - Maria Jose Rueda • \\ Baojin Zhu $\cdot$ Brett King
}

Received: May 5, 2021 / Accepted: June 23, 2021 / Published online: July 22, 2021

(C) The Author(s) 2021

\begin{abstract}
Introduction: Alopecia areata (AA) is an autoimmune disorder causing sudden, nonscarring hair loss. There are currently no drugs approved for AA treatment. This study assessed prevalence of comorbidities, treatments, and healthcare costs and resource utilization among patients with AA in the USA.

Methods: Patients diagnosed with AA between January 2011 and December 2018 were identified in IBM MarketScan ${ }^{\circledR}$ Research Databases. Eligible patients had no other hair loss-related disorders and were continuously enrolled with medical and pharmacy benefits at least 12 months before and after AA diagnosis.
\end{abstract}

Supplementary Information The online version contains supplementary material available at https:// doi.org/10.1007/s12325-021-01845-0.

M. Senna $(\bowtie)$

Massachusetts General Hospital, Boston, MA, USA

e-mail:msenna@partners.org

J. Ko

Stanford University, Stanford, CA, USA

A. Tosti

University of Miami, Miami, FL, USA

E. Edson-Heredia - D. C. Fenske - A. K. Ellinwood .

M. J. Rueda - B. Zhu

Eli Lilly and Company, Indianapolis, IN, USA

B. King

Yale School of Medicine, New Haven, CT, USA
Descriptive statistics were used to summarize comorbid conditions, treatments related to AA or other autoimmune/inflammatory conditions, and all-cause and AA-specific healthcare costs and resource utilization identified from claims data.

Results: A total of 68,121 patients with AA were identified. Mean (SD) age was 40.3 (17.8) years and $61.0 \%$ were female. The most common comorbidities included hyperlipidemia (22.4\%), hypertension $(21.8 \%)$, thyroid disorders $(13.1 \%)$, contact dermatitis or eczema $(10.8 \%)$, depression $(9.5 \%)$, and anxiety $(8.4 \%)$. Comorbid autoimmune diseases included atopic dermatitis $(2.8 \%)$, psoriasis $(2.1 \%)$, chronic urticaria (1.5\%), and rheumatoid arthritis (1.1\%). During the 12-month follow-up period, 37,995 patients $(55.8 \%)$ were prescribed treatment for their AA or other comorbid autoimmune/inflammatory disease; $44.9 \%$ of treated patients were prescribed therapy within 7 days of AA diagnosis. Of patients receiving treatment, $80.3 \%$ received topical steroids and $30.0 \%$ received oral steroids. Mean (SD) total healthcare costs were $\$ 11,241.21(\$ 43,839.69)$ for all-causes and $\$ 419.12$ (\$1534.99) for AA. AA-related expenses were driven by outpatient and prescription costs.

Conclusion: Patients with AA have a high comorbidity burden and lack of treatment. Current AA treatments, including systemic therapies other than oral steroids, were not frequently utilized in this study population. 
Healthcare costs incurred by patients with AA went beyond AA-related expenses. Longitudinal data are needed to better understand treatment trajectories and the disease burden in patients with AA.

Keywords: Alopecia areata; Comorbidity; Healthcare costs; Healthcare utilization; Treatment

\section{Key Summary Points}

Why carry out this study?

There are currently no drugs approved by the US Food and Drug Administration for the treatment of alopecia areata (AA).

To better understand treatment needs and burden in AA, this study assessed the prevalence of comorbidities, treatment patterns, and healthcare costs in patients with AA.

\section{What was learned from the study?}

Of 68,121 patients with AA, $55.8 \%$ were prescribed treatment for AA within a year of diagnosis.

Existing off-label treatments for AA, including systemic therapies other than oral steroids, were not frequently utilized in this study population, underscoring the need for effective treatment options to manage this disease. their first hair loss episode before the age of 40 , but AA can occur at any age and has a lifetime risk of nearly $2 \%$ worldwide $[2,4]$. AA is unpredictable, with spontaneous hair regrowth occurring in an estimated $34-50 \%$ of patients within the first year [2], though many will experience repeat episodes and can relapse at any time [5].

Alopecia areata often co-occurs with other autoimmune diseases and psychiatric disorders and can have serious impacts on patients' quality of life and psychological well-being [6-8]. However, there are currently no drugs approved by the US Food and Drug Administration (FDA) for the treatment of AA, resulting in a large unmet medical need. Existing off-label treatments for AA, including intralesional steroids for mild disease and topical and/or oral steroids for more severe cases, have limited effectiveness [9-11]. Given the limited treatment options, management of AA is difficult and can be burdensome to patients.

A better understanding of how current offlabel treatments are utilized in the AA population, as well as the economic impact of AA care in these patients and co-occurrence of psychiatric and medical conditions, can provide important insight into treatment needs and burden in AA. Using administrative claims data, this analysis seeks to assess the prevalence of comorbidities, evaluate treatment patterns, and describe costs of care in patients diagnosed with $\mathrm{AA}$ in the USA.

\section{METHODS}

\section{Data Source and Study Population}

\section{INTRODUCTION}

Alopecia areata (AA) is a chronic autoimmune disorder causing sudden, non-scarring hair loss. Prevalent in about $0.21 \%$ of the US population [1], the disorder is heterogenous in severity and distribution and can affect any hair-bearing region of the body [2]. Patients may progress from patchy AA to complete scalp hair loss (alopecia totalis) or complete body hair loss (alopecia universalis) [2, 3]. Patients often have
This retrospective, observational claims analysis utilizes data from the IBM MarketScan ${ }^{\circledR}$ Commercial Claims and Encounters Database (Commercial) and the Medicare Supplemental and Coordination of Benefits Database (Medicare Supplemental). These research databases contain detailed, patient-level inpatient, outpatient, and outpatient prescription drug encounters of over 200 million people in the USA who receive care under fee-for-service and managed care plans, including exclusive 
provider organizations (EPO), preferred provider organizations (PPO), point-of-service (POS) plans, indemnity plans, and health maintenance organizations (HMOs). The databases contain standard codes for diagnoses, procedures, and medications, and all claims in the research databases are fully paid and adjudicated. Member identification codes allow patients to be followed longitudinally [12].

Study subjects included those enrolled in the MarketScan databases who had at least one outpatient visit, inpatient admission, or healthcare provider visit with an AA diagnosis (International Classification of Diseases, Ninth Revision, Clinical Modification [ICD-9-CM] diagnosis codes 704.01 or 704.09 , or Tenth Revision [ICD-10-CM] diagnosis codes L63.0, L63.1, L63.2, L63.8, or L63.9) between January 1, 2011 and December 31, 2018. The index date was defined as the date of the first observed AA diagnosis. Patients must have been continuously enrolled with medical and pharmacy benefits for at least 12 months before the index date (baseline period) and at least 12 months after the index date (follow-up period). Excluded from this analysis were patients with other hair loss disorders, including trichotillomania, androgenic alopecia, telogen effluvium, tinea capitis and tinea barbae, scarring alopecia, unspecified non-scarring hair loss, pseudopelade, folliculitis decalvans, and other specified hair loss.

This study was conducted in accordance with ethical principles of the Declaration of Helsinki and Good Clinical Practice guidelines. This study was exempt from informed consent requirements and institutional/ethical review board approval was not required because this was a non-interventional study based on secondary data use. All patient data were de-identified and compliant with the Health Insurance Portability and Accountability Act of 1996.

\section{Measures and Outcomes}

\section{Demographics and Clinical Characteristics}

Demographics measured on the index date included age, sex, geographic region, and primary payer (Commercial or Medicare). Newly diagnosed AA was defined as having a diagnosis of AA during the follow-up period (including the index date) and no diagnosis of AA during the baseline period. Severity of AA was determined using diagnosis codes and/or prescription treatments as a proxy measure. Patients with a diagnosis code for alopecia universalis (L63.0) or alopecia totalis (L63.1) or prescriptions for any immunomodulators, oral steroids, systemic non-steroids, or phototherapy were classified as having moderate-to-severe disease, while patients who were prescribed topical therapies, intralesional steroids, non-traditional treatments, or other treatments were categorized as having mild disease. The list of treatments is in the supplementary material (Table S1).

\section{Comorbidities}

Comorbid conditions were identified by the presence of at least one inpatient or non-diagnostic outpatient medical claim with an ICD-9$\mathrm{CM}$ and ICD-10-CM diagnosis code during the baseline and follow-up periods. Comorbidities were compared between the baseline versus follow-up periods and between mild versus moderate-to-severe disease.

\section{Treatments}

Medications and therapies related to AA or other autoimmune/inflammatory conditions included topical steroids, intralesional triamcinolone, oral steroids, phototherapy, immunomodulators, and alternative therapies (Table S1 in the supplementary material). Prescribed treatments were evaluated within 7 days of diagnosis with AA (index therapy) and during the first 12 months post-AA diagnosis (followup treatment).

\section{Healthcare Costs and Utilization}

All-cause and AA-specific healthcare resource utilization and costs were measured for inpatient, outpatient, and emergency room (ER) visits and outpatient prescriptions during the 12 months after diagnosis with AA. AA-related encounters and costs included those associated with inpatient claims with AA as the principal diagnosis, outpatient or ER claims with an AA 
diagnosis in the first position, or AA-related medications. The costs of services provided under capitated arrangements were estimated using payment proxies that were computed on the basis of paid claims at the procedure level. All costs were inflated to 2019 US dollars using the Medical Care Component of the Consumer Price Index.

\section{Statistical Analysis}

The study sample selection and creation of analytic variables were conducted using Instant Health Data (IHD), a Software as a Service-based real-world evidence analytics platform (Boston, MA, USA). Descriptive statistics were used to summarize the study population, comorbidity prevalence, treatments, and healthcare resource utilization and costs, with counts $(N)$ and percentages for categorical measures and means and standard deviations (SDs), medians and interquartile ranges (IQRs), and ranges (min-$\max )$ for continuous measures. Data were analyzed using R, version 3.2.1 (Vienna, Austria).

\section{RESULTS}

\section{Patient Characteristics}

A total of 68,121 patients with a diagnosis of AA were identified between 2011 and 2018 (Fig. S1 in the supplementary material). The mean (SD) age at the time of diagnosis was 40.3 (17.8) years and $61.0 \%$ of patients were female (Table 1 ). About half (53.8\%) were seen by a dermatologist and $96.4 \%$ were newly diagnosed with AA. A total of $10,305(15.1 \%)$ had mild AA, 23,730 (34.8\%) had moderate-to-severe AA, and 34,086 (50.0\%) had unknown/indeterminate severity. The diagnosis codes for alopecia totalis and alopecia universalis were identified in 900 patients (1.3\%) and 435 patients (0.6\%), respectively.

\section{Comorbidities}

The prevalence of comorbidities before and after diagnosis with $\mathrm{AA}$ is listed in Table 2. In
Table 1 Characteristics of patients with a diagnosis of alopecia areata $(N=68,121)$

\begin{tabular}{ll}
\hline Characteristic & $\boldsymbol{N}(\%)$ \\
\hline Age, mean (SD) & $40.3(17.8)$ \\
Gender & \\
Female & $41,561(61.0 \%)$ \\
Male & $26,560(39.0 \%)$ \\
Geographic region & \\
Midwest & $12,424(18.2 \%)$ \\
Northeast & $15,423(22.6 \%)$ \\
South & $26,220(38.5 \%)$ \\
West & $12,754(18.7 \%)$ \\
Unknown & $1300(1.9 \%)$ \\
Primary payer & \\
Commercial & $63,127(92.7 \%)$ \\
Medicare & $4987(7.3 \%)$ \\
Unknown & $7(0.01 \%)$ \\
Disease severity & \\
Mild & $10,305(15.1 \%)$ \\
Moderate-to-severe & $23,730(34.8 \%)$ \\
Unknown/indeterminate & $34,086(50.0 \%)$ \\
Diagnosed by a dermatologist & $36,620(53.8 \%)$ \\
New AA diagnosis & $65,678(96.4 \%)$ \\
\hline Disease severy was defned &
\end{tabular}

${ }^{a}$ Disease severity was defined using treatments and diagnosis as a proxy

general, the percentages of patients with comorbid conditions were higher in the followup period. The most common coexisting conditions during follow-up were hyperlipidemia $(22.4 \%)$, hypertension $(21.8 \%)$, thyroid disorders $(13.1 \%)$, contact dermatitis or eczema $(10.8 \%)$, depression $(9.5 \%)$, and anxiety $(8.4 \%)$. Comorbid autoimmune diseases included atopic dermatitis $(2.8 \%)$, psoriasis $(2.1 \%)$, chronic urticaria $(1.5 \%)$, and rheumatoid arthritis (1.1\%).

Comorbidities among the subset of 34,035 patients for whom disease severity was 
Table 2 Prevalence of comorbidities in patients with alopecia areata during the baseline and follow-up periods $(N=68,121)$

\begin{tabular}{|c|c|c|}
\hline Comorbidities & Baseline & Follow-up \\
\hline Hyperlipidemia & $13,904(20.4 \%)$ & $15,226(22.4 \%)$ \\
\hline Hypertension & $13,751(20.2 \%)$ & $14,858(21.8 \%)$ \\
\hline Thyroid disorder & $7549(11.1 \%)$ & $8949(13.1 \%)$ \\
\hline Contact dermatitis and eczema & $5379(7.9 \%)$ & $7324(10.8 \%)$ \\
\hline Depression & $5505(8.1 \%)$ & $6466(9.5 \%)$ \\
\hline Anxiety & $4452(6.5 \%)$ & $5718(8.4 \%)$ \\
\hline Obesity & $4216(6.2 \%)$ & $5236(7.7 \%)$ \\
\hline Asthma & $4489(6.6 \%)$ & $4690(6.9 \%)$ \\
\hline Osteoarthritis & $4125(6.1 \%)$ & $4375(6.4 \%)$ \\
\hline Diabetes mellitus & $4273(6.3 \%)$ & $4186(6.1 \%)$ \\
\hline Coronary heart disease & $2650(3.9 \%)$ & $2936(4.3 \%)$ \\
\hline Atopic dermatitis & $1352(2.0 \%)$ & $1932(2.8 \%)$ \\
\hline Tobacco dependency & $1441(2.1 \%)$ & $1733(2.5 \%)$ \\
\hline Psoriasis & $908(1.3 \%)$ & $1408(2.1 \%)$ \\
\hline Cerebrovascular disease & $1303(1.9 \%)$ & $1387(2.0 \%)$ \\
\hline Chronic urticaria & $851(1.3 \%)$ & $996(1.5 \%)$ \\
\hline Hypersensitivity & $829(1.2 \%)$ & $829(1.2 \%)$ \\
\hline Rheumatoid arthritis & $683(1.0 \%)$ & $750(1.1 \%)$ \\
\hline Vitiligo & $261(0.4 \%)$ & $561(0.8 \%)$ \\
\hline Alcohol abuse & $414(0.6 \%)$ & $478(0.7 \%)$ \\
\hline Suicidal ideation & $428(0.6 \%)$ & $472(0.7 \%)$ \\
\hline Systemic lupus erythematosus & $385(0.6 \%)$ & $460(0.7 \%)$ \\
\hline Ulcerative colitis & $310(0.5 \%)$ & $334(0.5 \%)$ \\
\hline Crohn's disease & $295(0.4 \%)$ & $282(0.4 \%)$ \\
\hline Sjogren's syndrome & $200(0.3 \%)$ & $247(0.4 \%)$ \\
\hline Multiple sclerosis & $192(0.3 \%)$ & $209(0.3 \%)$ \\
\hline Celiac disease & $144(0.2 \%)$ & $186(0.3 \%)$ \\
\hline Uveitis & $190(0.3 \%)$ & $165(0.2 \%)$ \\
\hline Hidradenitis suppurativa & $119(0.2 \%)$ & $154(0.2 \%)$ \\
\hline Psoriatic arthritis & $118(0.2 \%)$ & $136(0.2 \%)$ \\
\hline Ankylosing spondylitis & $53(0.1 \%)$ & $60(0.1 \%)$ \\
\hline Systemic sclerosis & $45(0.1 \%)$ & $60(0.1 \%)$ \\
\hline
\end{tabular}


Table 2 continued

\begin{tabular}{lll}
\hline Comorbidities & Baseline & Follow-up \\
\hline Tuberculosis & $43(0.06 \%)$ & $31(0.05 \%)$ \\
Reactive arthritis & $10(0.01 \%)$ & $8(0.01 \%)$ \\
\hline
\end{tabular}

Data presented as $n$ (\%). Categories are not mutually exclusive

estimated are summarized in Table 3. The prevalence of comorbid conditions was numerically higher among patients with more severe disease. In patients with moderate-to-severe and mild disease, respectively, thyroid disorders were prevalent in $14.7 \%$ and $9.7 \%$ of patients, depression was reported in $11.2 \%$ and $7.3 \%$ of patients, and anxiety was observed in $8.6 \%$ and $7.1 \%$ of patients.

\section{Treatments}

During the 12 months after diagnosis with AA, 37,995 (55.8\%) patients were prescribed treatment for their AA or other comorbid autoimmune/inflammatory disease, with 10,352 patients $(27.2 \%$ of treated patients, $15.2 \%$ overall) receiving at least two treatment types. Within 7 days of AA diagnosis, 17,062 patients (44.9\% of treated patients, $25.1 \%$ overall) were prescribed treatment. Overall, 30,126 (44.2\%) patients were not prescribed treatment in the year following diagnosis, including 29,071/ 65,678 patients $(44.3 \%)$ who were newly diagnosed with AA.

The distributions of treatments prescribed within 7 days and 12 months of AA diagnosis are listed in Table 4. Topical steroids and oral steroids, respectively, were given to $80.3 \%$ and $30.0 \%$ of treated patients throughout follow-up.

\section{Healthcare Costs and Utilization}

In the 12 months after diagnosis with $\mathrm{AA}$, mean (SD) total healthcare costs were $\$ 11,241.21$ $(\$ 43,839.69)$ for all-causes and $\$ 419.12$ (\$1534.99) for AA (Fig. 1). Out-of-pocket costs totaled \$1175.20 (1654.52) for all-causes and \$104.19 (201.20) for AA. Outpatient visits (\$226.17 [526.42]) and prescriptions (\$190.39 [1423.16]) were the main sources of AA-related expenses. About $71.7 \%$ and $55.3 \%$ of patients had outpatient and pharmacy visits, respectively, during the 12-month period after diagnosis (Table 5).

\section{DISCUSSION}

This comprehensive study of claims data sought to describe comorbidities, treatment patterns, and healthcare costs and utilization in patients diagnosed with AA in the USA. Comorbid conditions, including thyroid disorder, depression, anxiety, and other autoimmune diseases, were more commonly reported after AA diagnosis and in patients with moderate-to-severe disease. About $55.8 \%$ of patients were treated with offlabel treatments for AA or another autoimmune/inflammatory condition in the first year after AA diagnosis, with $44.9 \%$ of these patients being prescribed treatment within 1 week of their AA diagnosis. The most prescribed treatments were topical steroids, followed by oral steroids. Finally, AA-related healthcare expenditures were largely driven by outpatient and medication costs. Together, these findings can contribute to our understanding of treatment needs and burden in AA.

Hyperlipidemia and hypertension were the most prevalent comorbid conditions in this study population, with rates similar to those previously reported [13]. Depression and anxiety were also common in this cohort. Prior studies have found that rates of mental health 
Table 3 Prevalence of comorbidities in patients with alopecia areata by disease severity $(N=34,035)$

\begin{tabular}{|c|c|c|}
\hline Comorbidities & Mild $(N=10,305)$ & Moderate-to-severe $(N=23,730)$ \\
\hline Hypertension & $1786(17.3 \%)$ & $6242(26.3 \%)$ \\
\hline Hyperlipidemia & $1816(17.6 \%)$ & $6133(25.8 \%)$ \\
\hline Thyroid disorder & $999(9.7 \%)$ & $3484(14.7 \%)$ \\
\hline Contact dermatitis and eczema & $1081(10.5 \%)$ & $3110(13.1 \%)$ \\
\hline Depression & $750(7.3 \%)$ & $2649(11.2 \%)$ \\
\hline Obesity & $674(6.5 \%)$ & $2061(8.7 \%)$ \\
\hline Diabetes mellitus & $457(4.4 \%)$ & $2050(8.6 \%)$ \\
\hline Anxiety & $734(7.1 \%)$ & $2034(8.6 \%)$ \\
\hline Osteoarthritis & $439(4.3 \%)$ & $2028(8.6 \%)$ \\
\hline Asthma & $509(4.9 \%)$ & $1936(8.2 \%)$ \\
\hline Coronary heart disease & $300(2.9 \%)$ & $1248(5.3 \%)$ \\
\hline Atopic dermatitis & $451(4.4 \%)$ & $694(2.9 \%)$ \\
\hline Tobacco dependency & $236(2.3 \%)$ & $629(2.7 \%)$ \\
\hline Cerebrovascular disease & $150(1.5 \%)$ & $575(2.4 \%)$ \\
\hline Psoriasis & $253(2.5 \%)$ & $554(2.3 \%)$ \\
\hline Chronic urticaria & $113(1.1 \%)$ & $402(1.7 \%)$ \\
\hline Hypersensitivity & $62(0.6 \%)$ & $395(1.7 \%)$ \\
\hline Rheumatoid arthritis & $59(0.6 \%)$ & $389(1.6 \%)$ \\
\hline Systemic lupus erythematosus & $37(0.4 \%)$ & $223(0.9 \%)$ \\
\hline Suicidal ideation & $55(0.5 \%)$ & $193(0.8 \%)$ \\
\hline Alcohol abuse & $65(0.6 \%)$ & $162(0.7 \%)$ \\
\hline Vitiligo & $132(1.3 \%)$ & $154(0.7 \%)$ \\
\hline Ulcerative colitis & $29(0.3 \%)$ & $136(0.6 \%)$ \\
\hline Sjogren's syndrome & $8(0.1 \%)$ & $128(0.5 \%)$ \\
\hline Crohn's disease & $23(0.2 \%)$ & $127(0.5 \%)$ \\
\hline Multiple sclerosis & $19(0.2 \%)$ & $92(0.4 \%)$ \\
\hline Uveitis & $6(0.1 \%)$ & $79(0.3 \%)$ \\
\hline Hidradenitis suppurativa & $18(0.2 \%)$ & $70(0.3 \%)$ \\
\hline Psoriatic arthritis & $11(0.1 \%)$ & $68(0.3 \%)$ \\
\hline Celiac disease & $19(0.2 \%)$ & $66(0.3 \%)$ \\
\hline Systemic sclerosis & $4(0.0 \%)$ & $33(0.1 \%)$ \\
\hline Ankylosing spondylitis & $9(0.1 \%)$ & $26(0.1 \%)$ \\
\hline
\end{tabular}


Table 3 continued

\begin{tabular}{lll}
\hline Comorbidities & Mild $(\boldsymbol{N}=\mathbf{1 0 , 3 0 5})$ & Moderate-to-severe $(\boldsymbol{N}=\mathbf{2 3 , 7 3 0})$ \\
\hline Tuberculosis & $1(0.0 \%)$ & $13(0.1 \%)$ \\
Reactive arthritis & $0(0.0 \%)$ & $4(0.0 \%)$ \\
\hline
\end{tabular}

Data presented as $n$ (\%). Categories are not mutually exclusive

Table 4 Treatments prescribed to patients with alopecia areata within 12 months of diagnosis

\begin{tabular}{lll}
\hline Treatment class & Index treatment $^{\mathbf{a}}(\boldsymbol{N}=\mathbf{1 7 , 0 6 2})$ & Follow-up treatment $(\boldsymbol{N}=\mathbf{3 7 , 9 9 5})$ \\
\hline Topical steroids & $14,804(86.8 \%)$ & $30,526(80.3 \%)$ \\
Oral steroids & $801(4.7 \%)$ & $11,394(30.0 \%)$ \\
Systemic antihistamines & $309(1.8 \%)$ & $2346(6.2 \%)$ \\
Topical non-steroids $^{\mathrm{b}}$ & $701(4.1 \%)$ & $2172(5.7 \%)$ \\
Finasteride & $723(4.2 \%)$ & $1432(3.8 \%)$ \\
Immunomodulator $^{\mathrm{c}}$ & $116(0.7 \%)$ & $1367(3.6 \%)$ \\
Acupuncture & $100(0.6 \%)$ & $630(1.7 \%)$ \\
Phototherapy & $129(0.8 \%)$ & $295(0.8 \%)$ \\
Systemic non-steroids & $37(0.2 \%)$ & $127(0.3 \%)$ \\
Intralesional triamcinolone $^{\text {Platelet-rich plasma }}$ & $9(0.1 \%)$ & $32(0.1 \%)$ \\
\hline
\end{tabular}

Data presented as $n$ (\%). Some patients received two or more treatment types

${ }^{a}$ Index treatment: treatment prescribed to patients within 7 days of AA diagnosis. The index treatment group is a subset of the broader follow-up treatment group

b Pimecrolimus, tacrolimus, calcipotriene, calcipotriene and betamethasone dipropionate, minoxidil, anthralin or dithranol, and topical antihistamines

c Methotrexate, azathioprine, cyclosporine, sulfasalazine, tofacitinib, baricitinib, apremilast, ruxolitinib, dupilumab, secukinumab, ixekizumab, brodalumab

disorders are higher in patients with AA versus controls without AA $[14,15]$. Psychiatric conditions may be both contributors to and consequences of AA $[14,16]$, underscoring the significance of these disorders in patients with this disease. Thyroid and other autoimmune disorders were also common among patients with alopecia in this analysis. Many studies suggest an association between AA and autoimmune conditions, including psoriasis, systemic lupus erythematosus, vitiligo, and atopic dermatitis [13, 17]. Awareness of these potential comorbidities is important for therapeutic management of AA [18].

In this study, $55.8 \%$ of patients received prescription treatment in the 12 -month period following diagnosis with $\mathrm{AA}$, with $25.1 \%$ of all patients being prescribed treatment within 1 week of diagnosis. The apparent delay in prescribing treatment likely reflects both watchful waiting in some patients, as spontaneous hair regrowth occurs in about half of mild cases [19], 
All Causes

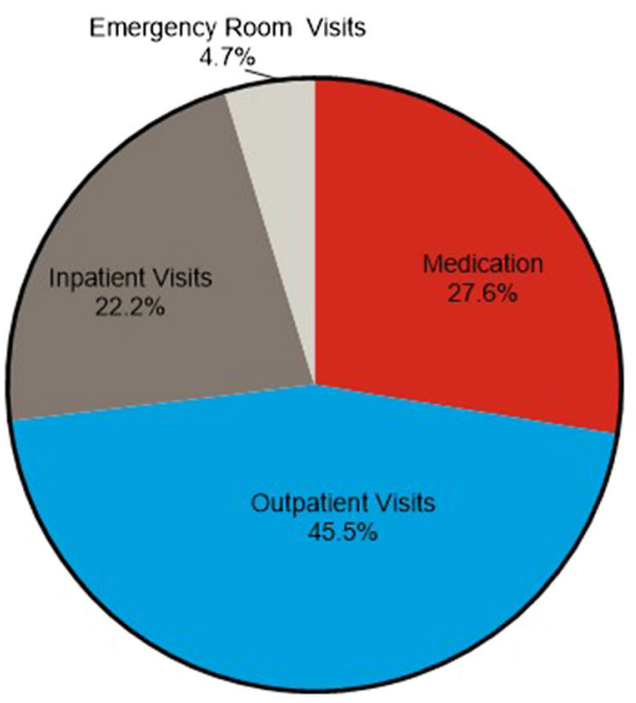

Alopecia Areata

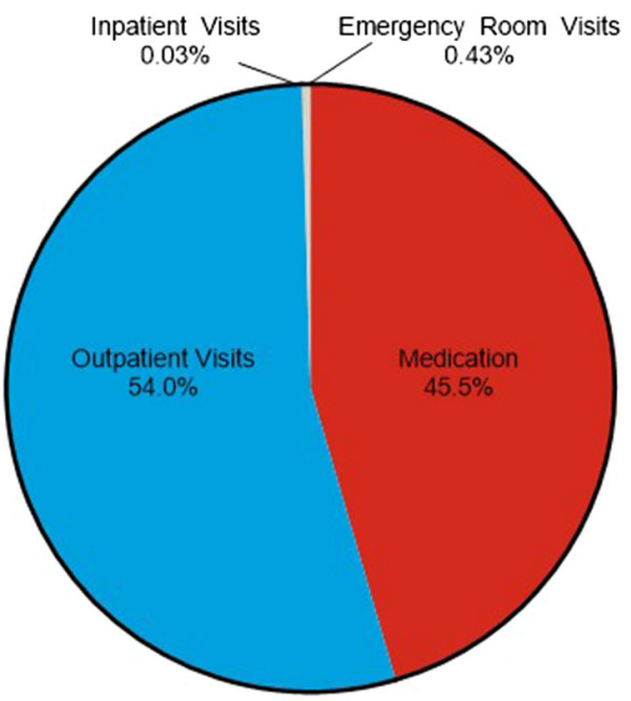

All Causes

Alopecia Areata

\begin{tabular}{|c|c|c|}
\hline Healthcare Costs & & \\
\hline Total & $\$ 11,241.21(43,839.69)$ & $\$ 419.12(1,534.99)$ \\
\hline Net Payment & $\$ 10,020.43(43,378.25)$ & $\$ 312.97(1,473.84)$ \\
\hline Out-of-Pocket & $\$ 1,175.20(1,654.52)$ & $\$ 104.19(201.20)$ \\
\hline Medical Costs & & \\
\hline Total & $\$ 8,153.27(37,806.79)$ & $\$ 228.73(544.12)$ \\
\hline Inpatient Visits & $\$ 2,480.05(29,054.83)$ & $\$ 0.14(35.75)$ \\
\hline Net Payment & $\$ 2,404.56(29,120.02)$ & $\$ 0.12(31.04)$ \\
\hline Out-of-Pocket & $\$ 72.52(491.68)$ & $\$ 0.02(4.71)$ \\
\hline Outpatient Visits & $\$ 5,099.48(17,989.76)$ & $\$ 226.17(526.42)$ \\
\hline Net Payment & $\$ 4,341.78(17,563.73)$ & $\$ 147.80(471.29)$ \\
\hline Out-of-Pocket & $\$ 723.27(1,044.60)$ & $\$ 74.88(146.53)$ \\
\hline Emergency Room Visits & $\$ 530.84(2,474.77)$ & $\$ 1.81(76.39)$ \\
\hline Net Payment & $\$ 450.72(2,316.10)$ & $\$ 1.63(76.58)$ \\
\hline Out-of-Pocket & $\$ 78.01(345.17)$ & $\$ 0.26(11.06)$ \\
\hline Medication Costs & & \\
\hline Total & $\$ 3,087.94(16,525.16)$ & $\$ 190.39(1,423.16)$ \\
\hline
\end{tabular}

Fig. 1 Proportion of healthcare spending for all causes and alopecia areata in the 12 months after diagnosis

and worsening of disease in other patients, as up to a quarter of patients progress to alopecia totalis or alopecia universalis [2].

Most treated patients were prescribed topical steroids, which may be beneficial in managing mild disease. However, topical steroids may not be beneficial in the long term and are less effective in treating more severe types of alopecia [2]. Oral steroids have demonstrated efficacy in stimulating hair regrowth in many cases of AA [10]. Intralesional steroids are considered the standard of care for patchy AA of limited extent [10]. In addition, immunosuppressive and immunotherapy drugs have been found to be somewhat effective in treating alopecia [17]. However, these treatments were not frequently utilized in the study population.

About $44 \%$ of patients in this study did not receive any prescription treatment for AA. These patients may, in part, represent those with mild 
Table 5 Healthcare resource utilization in patients with alopecia areata within 12 months after diagnosis $(N=68,121)$

\begin{tabular}{lll}
\hline Healthcare resource & All-cause & Alopecia areata \\
\hline Inpatient visits & & \\
$N$ (\%) & $4892(7.2 \%)$ & $1(0 \%)$ \\
Mean (SD) & $0.13(0.8)$ & $0(0)$ \\
Median (IQR) & $0(0-0)$ & $0(0-0)$ \\
Min-max & $0-64$ & $0-1$ \\
Length of inpatient visits & \\
Mean (SD) & $3.20(7.5)$ & $1(0)$ \\
Median (IQR) & $2(0-4)$ & $1(1-1)$ \\
Min-max & $0-278$ & $1-1$ \\
Outpatient visits & & \\
$N$ (\%) & $68,111(100 \%)$ & $48,847(71.7 \%)$ \\
Mean (SD) & $13.79(16.9)$ & $1.39(1.8)$ \\
Median (IQR) & $9(5-17)$ & $1(0-2)$ \\
Min-max & $0-633$ & $0-62$ \\
ER visits & & $1.57(2.7)$ \\
$N$ (\%) & $10,732(15.8 \%)$ & $69(0.10 \%)$ \\
Mean (SD) & $0.22(0.65)$ & $0(0.03)$ \\
Median (IQR) & $0(0-0)$ & $0(0-0)$ \\
Min-max & $0-26$ & $0-1$ \\
Pharmacy visits & & $1(0-2)$ \\
$N$ (\%) & $61,418(90.2 \%)$ & $37,665(55.3 \%)$ \\
Mean (SD) & $15.75(20.0)$ & $0-68$ \\
Median (IQR) & $9(3-21)$ & \\
Min-max & $0-327$ & \\
\hline & & \\
& &
\end{tabular}

disease who are advised to take a wait-and-see approach, or who spontaneously remit and for whom medical treatment may not be appropriate [10]. In addition, many patients elect to self-treat with over-the-counter medications or conceal the condition with wigs or make-up $[7,20]$. Importantly, patients with severe disease may forgo treatment, as prognosis is poor and current treatment options are unlikely to be effective in long-term management of disease $[20,21]$.

In this study, AA-related expenses largely consisted of outpatient and prescription costs. Over half of patients had pharmacy visits and nearly three-quarters had outpatient visits related to their AA. Though AA-related costs were a relatively small proportion of total healthcare costs in this study, other studies suggest that AA care is financially burdensome to patients [22]. The comparatively low cost of AA care in this study may reflect the large number of patients who were not prescribed any treatment. Additionally, the AA costs in this analysis do not include other important expenditures for these patients, including headwear or cosmetic options [22]. These findings also do not capture the substantial financial impacts of potential psychological distress or work productivity loss [23].

This analysis has important limitations. The study population was limited to individuals in the USA with private health insurance and Medicare supplemental coverage, and therefore these findings may not be generalizable beyond commercially insured patients with AA. It was not possible to link prescription claims to a specific diagnosis; the inability to confirm that medications were prescribed for AA treatment could inflate treatment rates reported in this study. Claims data have limited clinical and diagnostic characteristics. Prescription treatments were used as a proxy for disease severity, which may not accurately represent disease and fails to capture severity for untreated patients. Nearly half of patients were diagnosed with AA by non-dermatologists, and AA diagnoses could not be confirmed with chart reviews. It is possible that certain comorbid conditions were misclassified; patients with atopic dermatitis likely also experience contact dermatitis or eczema, and their condition may have been coded as such.

\section{CONCLUSIONS}

This large, comprehensive analysis highlights the high rate of comorbidities and utilization of 
healthcare in the AA patient population. Current off-label methods for treating AA, including systemic therapies beyond oral steroids, were not frequently used in this study population. As there are presently no FDA-approved treatments for $\mathrm{AA}$, these findings underscore the need for effective long-term treatment options to manage disease. Healthcare costs associated with AA are largely due to outpatient and medication costs, though the economic impact of AA cannot be fully captured in claims data. Future longitudinal analyses may consider the AA treatment trajectory throughout the course of disease to better understand the sequence and timing of treatments given. Studies of non-prescription management of disease would also yield important information into the burden of disease not captured in claims data.

\section{ACKNOWLEDGEMENTS}

Funding. This study and the journal's Rapid Service and Open Access fees were funded by Eli Lilly and Company.

Authorship. All named authors meet the International Committee of Medical Journal Editors (ICMJE) criteria for authorship for this article, take responsibility for the integrity of the work as a whole, and have given their approval for this version to be published.

Authorship Contributions. EEH, DCF, MJR, and $\mathrm{BZ}$ contributed to the conceptualization and design of the study. DCF performed the statistical analysis, and BZ provided consultation and support. All authors contributed to the data interpretation, critically reviewed the manuscript, and approved the submitted version.

Disclosures. Maryanne Senna has served on advisory boards and/or have been a consultant for Arena Pharmaceuticals, Concert Pharmaceuticals Inc., Eli Lilly and Company, Pfizer Inc, and Follica, Inc. She is a clinical trial investigator for Concert Pharmaceuticals Inc., Eli Lilly and Company, and Follica, Inc. Dr. Ko has served on advisory boards and is a consultant and clinical investigator for Eli Lilly and Company he has served as a clinical investigator and/or consultant for AbbVie, Sanofi, Regeneron, Dermira, BMS and Arena Pharmaceuticals. $\mathrm{He}$ has received consulting fees from Eli Lilly and Company, Concert Pharmaceuticals, and Arena Pharmaceuticals. Antonella Tosti is a consultant for DS Laboratories, Monat Global, Almirall, Tirthy Madison, Leo Pharmaceuticals, Bristol Myers Squibb, and P\&G. Dr. Tosti is a compensated consultant/advisory board member for Eli Lilly, sponsor of the study. Brett King has served on advisory boards and/or is a consultant and/or is a clinical trial investigator for Aclaris Therapeutics Inc, Arena Pharmaceuticals, Bristol-Meyers Squibb, Concert Pharmaceuticals Inc, Dermavant Sciences Inc, Eli Lilly and Company, Incyte Corp, Pfizer Inc, TWi Biotechnology Inc, and Viela Bio. Emily Edson-Heredia, D. Christian Fenske, Amy K. Ellinwood, Maria Jose Rueda, and Baojin Zhu are employees and shareholders of Eli Lilly and Company.

Compliance with Ethics Guidelines. This study was conducted in accordance with ethical principles of the Declaration of Helsinki and Good Clinical Practice guidelines. This study was exempt from informed consent requirements, and institutional/ethical review board approval was not required because this was a non-interventional study based on secondary data use. All patient data were de-identified and compliant with the Health Insurance Portability and Accountability Act of 1996.

Data Availability. The data that support the findings of this study were provided by IBM. Restrictions apply to the availability of these data, which were used under license for this study and therefore not publicly available. Requests may be sent to IBM for more information on data availability and licensing.

Prior Presentation. Data from this study were presented in part at the Innovations in Dermatology Virtual Spring Conference held from March 16-20, 2021. 
Open Access. This article is licensed under a Creative Commons Attribution-NonCommercial 4.0 International License, which permits any non-commercial use, sharing, adaptation, distribution and reproduction in any medium or format, as long as you give appropriate credit to the original author(s) and the source, provide a link to the Creative Commons licence, and indicate if changes were made. The images or other third party material in this article are included in the article's Creative Commons licence, unless indicated otherwise in a credit line to the material. If material is not included in the article's Creative Commons licence and your intended use is not permitted by statutory regulation or exceeds the permitted use, you will need to obtain permission directly from the copyright holder. To view a copy of this licence, visit http://creativecommons.org/licenses/by$\mathrm{nc} / 4.0 /$.

\section{REFERENCES}

1. Benigno $\mathrm{M}$, Anastassopoulos $\mathrm{KP}$, Mostaghimi A, et al. A large cross-sectional survey study of the prevalence of alopecia areata in the United States. Clin Cosmet Investig Dermatol. 2020;13:259-66.

2. Pratt CH, King LE Jr, Messenger AG, Christiano AM, Sundberg JP. Alopecia areata. Nat Rev Dis Primers. 2017;3:17011.

3. Betz RC, Petukhova L, Ripke S, et al. Genome-wide meta-analysis in alopecia areata resolves HLA associations and reveals two new susceptibility loci. Nat Commun. 2015;6:5966.

4. Villasante Fricke AC, Miteva M. Epidemiology and burden of alopecia areata: a systematic review. Clin Cosmet Investig Dermatol. 2015;8:397-403.

5. Lyakhovitsky A, Aronovich A, Gilboa S, Baum S, Barzilai A. Alopecia areata: a long-term follow-up study of 104 patients. J Eur Acad Dermatol Venereol. 2019;33(8):1602-9.

6. Abedini R, Hallaji Z, Lajevardi V, Nasimi M, Karimi Khaledi M, Tohidinik HR. Quality of life in mild and severe alopecia areata patients. Int J Womens Dermatol. 2018;4(2):91-4.

7. Aldhouse NVJ, Kitchen H, Knight S, et al. "'You lose your hair, what's the big deal?' I was so embarrassed, I was so self-conscious, I was so depressed:" a qualitative interview study to understand the psychosocial burden of alopecia areata. J Patient Rep Outcomes. 2020;4(1):76.

8. Liu LY, King BA, Craiglow BG. Health-related quality of life (HRQoL) among patients with alopecia areata (AA): a systematic review. J Am Acad Dermatol. 2016;75(4):806 e3-812 e3.

9. Juarez-Rendon KJ, Rivera Sanchez G, Reyes-Lopez MA, et al. Alopecia areata. Current situation and perspectives. Arch Argent Pediatr. 2017;115(6): e404-11.

10. Strazzulla LC, Wang EHC, Avila L, et al. Alopecia areata: an appraisal of new treatment approaches and overview of current therapies. J Am Acad Dermatol. 2018;78(1):15-24.

11. Meah N, Wall D, York K, et al. The Alopecia Areata Consensus of Experts (ACE) study: results of an international expert opinion on treatments for alopecia areata. J Am Acad Dermatol. 2020;83(1): 123-30.

12. IBM Watson Health. IBM MarketScan Research Databases for health services researchers. Somers: $2019 . \quad$ https://www.ibm.com/downloads/cas/ 6KNYVVQ2. Accessed 3 Nov 2020.

13. Huang KP, Mullangi S, Guo Y, Qureshi AA. Autoimmune, atopic, and mental health comorbid conditions associated with alopecia areata in the United States. JAMA Dermatol. 2013;149(7): 789-94.

14. Kim JC, Lee ES, Choi JW. Impact of alopecia areata on psychiatric disorders: a retrospective cohort study. J Am Acad Dermatol. 2020;82(2):484-6.

15. Singam V, Patel KR, Lee HH, Rastogi S, Silverberg JI. Association of alopecia areata with hospitalization for mental health disorders in US adults. J Am Acad Dermatol. 2019;80(3):792-4.

16. Dai YX, Tai YH, Chen CC, Chang YT, Chen TJ, Chen MH. Bidirectional association between alopecia areata and major depressive disorder among probands and unaffected siblings: a nationwide population-based study. J Am Acad Dermatol. 2020;82(5):1131-7.

17. Islam N, Leung PS, Huntley AC, Gershwin ME. The autoimmune basis of alopecia areata: a comprehensive review. Autoimmun Rev. 2015;14(2):81-9.

18. Lee $\mathrm{S}$, Lee $\mathrm{H}$, Lee $\mathrm{CH}$, Lee WS. Comorbidities in alopecia areata: a systematic review and metaanalysis. J Am Acad Dermatol. 2019;80(2):466 e16477 e16. 
19. Xu L, Liu KX, Senna MM. A practical approach to the diagnosis and management of hair loss in children and adolescents. Front Med (Lausanne). 2017;4:112.

20. Messenger AG, McKillop J, Farrant P, McDonagh AJ, Sladden M. British Association of Dermatologists' guidelines for the management of alopecia areata 2012. Br J Dermatol. 2012;166(5):916-26.

21. Rossi A, Muscianese M, Piraccini BM, et al. Italian guidelines in diagnosis and treatment of alopecia areata. G Ital Dermatol Venereol. 2019;154(6): 609-23.

22. Li SJ, Mostaghimi A, Tkachenko E, Huang KP. Association of out-of-pocket health care costs and financial burden for patients with alopecia areata. JAMA Dermatol. 2019;155(4):493-4.

23. Burns LJMN, Kranz D, Ellison A, Senna MM. Cumulative life course impairment of alopecia areata. Int J Trichol. 2020;12(5):197-204. 